\title{
Silicon-Based Hybrid Cell for Harvesting Solar Energy and Raindrop Electrostatic Energy
}

Li Zheng a,b,\#, Zong-Hong Lin ${ }^{\mathrm{a}, \# \text {, Gang Cheng }}{ }^{\mathrm{a}}$, Wenzhuo Wu ${ }^{\mathrm{a}}$, Xiaonan Wen ${ }^{\mathrm{a}}$, Sangmin Lee $^{\mathrm{c}}$, Zhong Lin Wang ${ }^{\mathrm{a}, \mathrm{d}, *}$

${ }^{a}$ School of Material Science and Engineering, Georgia Institute of Technology, Atlanta, Georgia 30332-0245, United States

${ }^{b}$ School of mathematics and physics, Shanghai University of Electric Power, Shanghai 200090, China

${ }^{c}$ School of Mechanical Engineering, Chung-Ang University, Seoul 156-756, Republic of Korea

${ }^{d}$ Beijing Institute of Nanoenergy and Nanosystems, Chinese Academy of Sciences, Beijing 100083, China

\#Authors contributed equally to this work.

*Corresponding author at: School of Materials Science and Engineering, Georgia Institute of Technology, Atlanta, Georgia, 30332-0245, United States

E-mail address: zlwang@gatech.edu (Z. L. Wang)

\begin{abstract}
Silicon-based solar cell is by far the most established solar cell technology. The surface of a Si solar cell is usually covered by a layer of transparent material to protect the device from environmental damages/corrosions. Here, we replaced this protection layer by a transparent triboelectric nanogeneartor (TENG), for simultaneously or individually
\end{abstract}


harvesting solar and raindrop energy when either or both of them are available in our living environment. The TENG is made of a specially processed polytetrafluoroethylene (PTFE) film, an indium tin oxide (ITO) and a polyethylene terephthalate (PET) layer. Under a solar light irradiation $\left(12 \mathrm{~W} / \mathrm{m}^{2}\right)$ in a rainy day, the fabricated high-efficiency solar cell provides an open-circuit $\left(V_{o c}\right)$ of $0.43 \mathrm{~V}$ and short-circuit current density $\left(J_{s c}\right)$ of 4.2 $\mathrm{A} / \mathrm{m}^{2}$. And the TENG designed for collection of raindrop energy gives an AC $V_{o c}$ of $30 \mathrm{~V}$ and $J_{s c}$ of $4.2 \mathrm{~mA} / \mathrm{m}^{2}$ when impacted by water drops at a dripping rate of $0.116 \mathrm{ml} / \mathrm{s}$. In rainy days, the performance of solar cell decreased greatly, while TENG can be a good compensation as for green energy harvesting. From these results, we can see that the hybrid cell formed by a solar cell and a water-drop TENG have great potential for simultaneously/individually harvesting both solar energy and raindrop electrostatic energy under different weather conditions, especially in raining season.

\section{Keywords}

hybrid cell; triboelectric nanogenerator; solar energy; raindrop electrostatic energy; energy harvesting;

\section{Introduction}

Energy crisis and environmental pollution have been the main challenges for a sustainable development of the world [1,2]. Harvesting energy from the natural environment such as solar [3,4], wind [5], biomass energy [6], mechanical vibration $[7,8]$ and wasted heat $[9,10]$, has attracted great interest in academic research in the past decades. Silicon based solar cells gradually become the most widely used commercial 
technology for large scale harvesting of solar energy because of its low cost and superior performance [11]. However, low conversion efficiency is still the main limiting factor for current technologies. A large portion of energy loss during solar-cell operation is attributed to the loss of incoming light by reflection [12]. To reduce the reflection loss, surface texturing [13-15] such as fabrication of various surface pyramid structures is often used to enhance light trapping by multiplying the internal reflections[13]. On the other hand, the solar cell panels have to be placed in ambient harsh environment with contaminations, corrosions, and varying weather conditions. In sunny days, solar cells can provide much electricity through photovoltaic effects. But in rainy days/season and night, the output of solar cells maybe largely suppressed or even vanished.

Currently, triboelectric nanogenerator (TENG) based on contact electrification and electrostatic induction effect has been developed to convert various forms of mechanical energy in the environment into electricity [16-21]. When two material surfaces with opposite triboelectric polarities are periodically contacted and separated, a periodic potential difference between the two electrodes on their backs varies, which drives electrons to flow through the external circuit and generate an AC electrical output [22]. Recently, triboelectrification on the water/solid [23] and water/air [24] interfaces are used to develop a new type of TENG, which demonstrates promising applications for harvesting energy from ocean wave, tide, and rain water in the environment $[25,26]$.

In practical applications, the surface of a solar cell is usually covered by a layer of transparent material to protect the device from environmental damages/corrosions. Since a water-drop TENG can be fabricated using highly transparent polymer materials, we could just replace the regular protection layer on solar panel using a specially designed 
and processed transparent polymer layer, to achieve the dual function of being a protection layer and a TENG. In this paper, we demonstrated a hybrid energy cell that consists of a specially designed micropyramidal Si solar cell and a water-drop TENG for simultaneously/individually harvesting both solar and raindrop electrostatic energy.

\section{Experimental Section}

\section{A. Fabrication of Si solar cell with micropyramids.}

The Si substrate used was a single-crystal, p-type float-zone substrate with a thickness of $300 \mu \mathrm{m}$. The textured Si surface with micropyramids was created by $\mathrm{KOH}$ etching. The wafer was then cleaned to remove surface organic and metallic contaminants, followed by $\mathrm{POCl}_{3}$ diffusion to form the $\mathrm{n}^{+}$-emitter. A diffusion temperature of $1133 \mathrm{~K}$ was used to obtain a $65 \Omega$ /sq emitter. The wafer was then coated with $80 \mathrm{~nm} \mathrm{SiN} \mathrm{via} \mathrm{a}$ plasma-enhanced chemical vapor deposition reactor. The $\mathrm{SiN}$ film serves as a passivation and antireflection coating layer for the device. After that, the screen-printed $\mathrm{n}^{+}-\mathrm{p}-\mathrm{p}^{+}$ junction solar cell was fabricated. An Al paste was screen-printed on the backside of the Si substrate and dried at $473 \mathrm{~K}$. Ag grids were then screen-printed on top of the $\mathrm{Si}$ substrate, followed by cofiring of both the Ag and Al contacts. An ITO top electrode with $300 \mathrm{~nm}$ thickness was coated by PVD 75 RF sputter.

\section{B. Fabrication of the superhydrophobic PTFE thin film.}

First, microstructures were fabricated by blasting an Al foil with sand particles using compressed air. The sand-blasted $\mathrm{Al}$ foil was further anodized in a $0.3 \mathrm{M}$ oxalic acid solution to obtain an anodic $\mathrm{Al}$ oxide (AAO) template with nanometer-sized holes. An SEM image of the AAO template is shown in Figure S2, where the average diameter of the holes in AAO template is about $46 \mathrm{~nm}$. Then after using a self-assembled monolayer 
of a heptadecafluoro-1,1,2,2-tetrahydrodecyl trichlorosilane (HDFS) to lower the surface energy of the AAO template, PTFE solvent was poured into the AAO template and a conventional vacuum process was applied to remove the air remaining in the nanoholes. After curing at the ambient temperature for one day, the solvent was evaporated and leaved a PTFE thin film with hierarchical nanostructures. Finally, the PTFE thin film was peeled off from the AAO template by using a transparent double-sided tape.

\section{Fabrication of hybrid cell.}

A commercial ITO film with thickness of $100 \mathrm{~nm}$ and PET thin layer was used as the electrode for water TENG, and then a PTFE thin film was attached on the ITO surface, thus a water TENG was fabricated. Then the fabricated water TENG was put on the surface of Si-based solar cell. Finally, some PDMS mixture with elastomer and crosslinker (Sylgard 184, Dow corning) mixed in a 10:1 mass ratio was used to seal all the electrodes of the device to prevent it from water.

\section{Measurement of hybrid cell.}

The solar cell efficiency was characterized under the light illumination intensity of $1000 \mathrm{~W} / \mathrm{m}^{2}$. The I-V curves of Si-based solar cell before and after integrating the waterdrop TENG was measured by using a Keithley 4200 semiconductor characterization system. The spectra transmittance of the TENG was measured by a UV-vis spectrophotometer (V-630). In the electric output measurement of the TENG, the water drops were sprayed by a shower which was connected to a household faucet and the water dripping rate was controlled by the knob of the shower. The current meter (SR570 low noise current amplifier, Stanford Research System) and voltage meter (6514 system electrometer, Keithley) were used to measure the electric output of the water-drop TENG. 


\section{Results and Discussion}

A schematic diagram of the fabricated hybrid energy cell is shown in Figure 1a, which is a fully integrated device constructed by a Si-based solar cell and a water-drop TENG. The bottom part is a Si-based solar cell with micropyramids composed of an Al film electrode, $\mathrm{a} \mathrm{p}^{+}$back surface field layer, a p-type Si bulk layer, a $\mathrm{n}^{+}$emitter layer, $\mathrm{SiN}$ film, Ag grids, and an ITO film electrode [13]. A photography of the fabricated Si solar cell is shown in Figure S1. The micropyramid surface morphology is designed to enhance absorption and suppress reflectance of the incoming light. This part can also be any kind of solar cell depending on application. The top part is a single-electrode-based water-drop TENG. The superhydrophobic PTFE thin film was fabricated by using a homemade porous anodic $\mathrm{Al}$ oxide (AAO) template, where the average diameter of the holes is about $46 \mathrm{~nm}$ (Figure S2). The detailed fabrication process of the Si solar cell, water-drop TENG and hybrid energy cell are described in the experimental section. Figure $1 \mathrm{~b}$ shows a scanning electron microscopy (SEM) image of the Si solar cell surface, indicating that the surface is micropyramidal with sizes from 1 to $10 \mu \mathrm{m}$. Figure 1c shows a SEM image of the prepared PTFE film. It is clearly shown that the surface of the PTFE film is composed of high-density nanorods. The average length and diameter of the nanorods are around $365 \mathrm{~nm}$ and $46 \mathrm{~nm}$, respectively. The high-density nanorod structures will contain trapped air and reduce the actual contact area between the surface and water, contributing to superhydrophobicity [27]. The inset in Figure 1c shows a incident angle of $169^{\circ}$ of the fabricated PTFE film, confirming the surface is superhydrophobic $\left(>150^{\circ}\right)$ [28].

The J-V curves in Figure 1d show the output performance of the fabricated Si solar cell before and after integrating with the water-drop TENG. Under the solar light irradiation 
$\left(1000 \mathrm{~W} / \mathrm{m}^{2}\right)$, the open-circuit voltage $\left(\mathrm{V}_{\mathrm{oc}}\right)$ and the short-circuit current density $\left(\mathrm{J}_{\mathrm{sc}}\right)$ of the Si solar cell are around $0.6 \mathrm{~V}$ and $350 \mathrm{~A} / \mathrm{m}^{2}$, respectively. The corresponding energy conversion efficiency is up to $16 \%$, which is much higher than the reported Si nanowire heterojunction solar cells [11]. The spectra transmittance of the water-drop TENG is shown in Figure S3, which clearly indicates that the water-drop TENG is highly transparent and nearly has no light absorption in the visible light region. After integrating the water-drop TENG on the top, the conversion efficiency of the solar cell under the same solar light irradiation was found to have a little decrease from $16 \%$ to $14 \%$. Although this slight drop in efficiency is not favored, it is an indispensable process in real applications because the silicon surface has to be covered by a layer of transparent thin film for protection purpose any way. Our design here is simply replacing the cover layer by a transparent TENG without affecting the performance of the original solar cell. It is worth noting that the superhydrophobicity of PTFE thin film will also enhance the automatically cleaning effect of solar cell surface, which will clean some particles falling on the surface and always provide a transparent protection layer, maintaining the standard conversion efficiency of the solar cell.

The working mechanism of the water-drop TENG is based on a sequential process of contact-electrification and electrostatic induction (Figure 2). For the purpose of applying this technique in real life, we design such a water-drop TENG to be operated in a singleelectrode mode [29]. As previous studies have pointed out, when a water drop falls from the sky or flows through an insulating tube, it will generate electrostatic charges due to the contact electrification with air, tube, or drifting particles [30-32]. The charges on the water drop surface could be positive or negative depending on the materials that the water 
drop interacted with. For simplification, we randomly choose several positively charged water drops to illustrate the dynamic process of energy transformation. As the positively charged water drops are reaching the PTFE film, a positive electric potential is established between the ITO electrode of PTFE film and the ground. In the short-circuit case, the electric potential difference (EPD) will drive the electrons transferring from the ground to the ITO electrode and thus generate an instant positive current until the EPD is decreased to zero. When the water drops leave the PTFE film with their positive tribocharges, a negative electric potential is created between the ITO electrode and the ground. As a consequence, the electrons will flow from the ITO electrode to the ground and generate an instant negative current until the EPD becomes zero. Therefore, the charge density on the water drop surface is a key factor to determine the magnitude of the electric output. If the water drops contact and leave the PTFE film periodically, continuous electric output can be provided.

To measure the electric output of the water-drop TENG, a shower nozzle is connected to a household faucet to spray water drops to simulate raindrops. The distance between the outlet of water drops and the TENG surface is $40 \mathrm{~cm}$. And the water dripping rate is controlled at $0.116 \mathrm{ml} / \mathrm{s}$. The typical output of $\mathrm{V}_{\mathrm{oc}}$ and $\mathrm{J}_{\mathrm{sc}}$ curves of the water-drop TENG are shown in Figures $3 a$ and $3 b$, and their magnification curves are shown in the insets. The $\mathrm{V}_{\mathrm{oc}}$ and $\mathrm{J}_{\mathrm{sc}}$ values of the TENG are around $30 \mathrm{~V}$ and $4.2 \mathrm{~mA} / \mathrm{m}^{2}$, respectively. There is a random fluctuation of the electric output of TENG in Figures $3 a$ and $3 b$. It is due to the fact that for plenty of water drops which fall randomly on the PTFE film surface, not all the drops can reach or leave the PTFE film simultaneously. The positive $\mathrm{V}_{\mathrm{oc}}$ value of the TENG indicates that the water drops flowing from the household faucet are positively 
charged, which is in good accordance with our previous report [24]. As the currently proposed working mechanism indicates, the positive and negative current peaks correspond to the processes of the contact and leave of the positively charged water drops on the TENG surface [25], respectively. From the inset of Figure 3b, we can observe that the positive current peak is higher than the negative current peak, revealing the removal process of the water drops on TENG surface requires a longer time than that of the contact process. This is due to the fact that the water drops in air fall freely onto the surface while the drops on the surface need slide to drip off, while sliding takes a longer time than free falling. Under the same experimental conditions, the output $\mathrm{J}_{\mathrm{sc}}$ of waterdrop TENG decreased sharply when replacing the PTFE film with a hydrophilic nylon film (Figure S4). This result shows that superhydrophobic surface plays a significant role for the prompt removal of the water drops on TENG surface and can enhance the electric output of water-drop TENG.

For a comprehensively characterization of the demonstrated water-drop TENG as a power source, the output voltage and current density of the water-drop TENG at various load resistances ranging from $10 \Omega$ to $1 \mathrm{G} \Omega$ were measured. And the corresponding output power density at each resistance was calculated using $P / A=I_{\text {load }} V_{\text {load }} / A=J_{\text {load }} V_{\text {load, }}$, where $\mathrm{A}$ is the surface area of the water-drop TENG and $\mathrm{J}_{\text {load }}$ is the current density generated from water TENG. As shown in Figure 3c, at the range below 0.1 M $\Omega$, both output voltage and current density only have little variation from the short-circuit condition. Then, an increase of the resistance beyond $1 \mathrm{M} \Omega$ will lead to the increase of the voltage and the decrease of the current density. Therefore, the instantaneous output power density on the load remains small when the resistance is below $0.1 \mathrm{M} \Omega$ and 
reaches the maximum value of $170 \mathrm{~mW} / \mathrm{m}^{2}$ at a resistance of $10 \mathrm{M} \Omega$ (Figure $3 \mathrm{~d}$ ).

We design such a hybrid energy cell aiming to apply it in real practical application on the widely used roof solar panels, so the influence of incident angle on the output performance of water-drop TENG is highly worth investigating, where the incident angle $\theta$ is between water dripping direction and TENG surface (inset of Figure 4a). Figures 4a and Figure $4 \mathrm{~b}$ show the measured $\mathrm{J}_{\mathrm{sc}}$ and $\mathrm{V}_{\mathrm{oc}}$ of the water-drop TENG at different incident angle ranging from 10 to 80 degree, respectively. The summarized relationships between $J_{\mathrm{sc}}, \mathrm{V}_{\mathrm{oc}}$ and the incident angle $\theta$ are shown in Figures $4 \mathrm{c}$ and $4 \mathrm{~d}$. When the incident angle was set from 10 to 45 degree, the measured output $\mathrm{J}_{\mathrm{sc}}$ and $\mathrm{V}_{\mathrm{oc}}$ changed from $1.7 \mathrm{~mA} / \mathrm{m}^{2}$ and $5.7 \mathrm{~V}$ to $4.2 \mathrm{~mA} / \mathrm{m}^{2}$ and $21.6 \mathrm{~V}$, respectively. With the increase of incident angle, $\mathrm{J}_{\mathrm{sc}}$ and $\mathrm{V}_{\mathrm{oc}}$ first increase linearly until $\theta$ reaches 30 degree, then keep constant until $\theta$ reaches 60 degree, and then decrease linearly with the increase of $\theta$. When $\theta$ is small, the effective contact surface area between water drops and TENG is small in the same experimental conditions, thus the amount of water drops falling on TENG surface in unit time is relatively small, which leads to small output $\mathbf{J}_{\mathrm{sc}}$ and $\mathrm{V}_{\mathrm{oc}}$ of TENG. On the other hand, with a large incident angle such as 80 degree, water drops that have falled on the TENG surface can not flow away from the surface quickly due to the nearly horizontal TENG surface, which will leave a thin layer of water in some area of the surface and this layer will screen the electrostatic induction effect between the charged water drops and the ground to a certain extent. So the output current and voltage decreased when $\theta$ was set at more than 60 degree. Due to the relatively large contact area and largely tilted degree for the water drops to slide away from the surface, the angle range of 30 60 degree is suitable for good output performance of water-drop TENG. The 
experimental results here reveal that water-drop TENG should be fixed at a certain tilted angle to obtain good output performance. On the other hand, previous studies have shown that roof solar panels are always installed at a certain tilted degree to capture much more incoming sunlight, which is determined by the latitude of location [33,34]. To obtain an optimal output of the designed hybrid cell which is constituted by solar cell and waterdrop TENG, the requirements for tilted angle in both solar cell panel and TENG is in good accordance, indicating that water-drop TENG can be integrated with solar cell panels in real industrial application in the near future.

To investigate the relationship between the output performance of the water-drop TENG with rainfall precipitation, water drops sprayed from a shower is used to simulate the raindrops. Figure 5a shows the output current density of the water-drop TENG generated under water impacting at different dripping rate from $0.064 \mathrm{ml} / \mathrm{s}$ to $0.116 \mathrm{ml} / \mathrm{s}$. With the increase of water dripping rate, the magnitude of current peaks decrease while the density of the current peaks increase. Considering the real physical process of current generation, when large amounts of water drops randomly fall onto and slide away from TENG surface, there will be some fluctuating compensation between the fall and removal of the charged water drops. As a result, the current peaks decrease while the peak density increase due to the increase of frequency of contact and removal of water drops on TENG surface. The instantaneous and average output power density of the water-drop TENG under different water dripping rate is calculated by $\mathrm{P} / \mathrm{A}=\mathrm{I}^{2} \mathrm{R} / \mathrm{A}$ and $\bar{P} / A=\frac{\int I^{2} R d t}{\Delta t \cdot A}$, and their corresponding curves are shown in Figures $5 \mathrm{~b}$ and Figure 5c, respectively. It is clearly shown from Figures $5 \mathrm{~b}$ and $5 \mathrm{c}$ that when water dripping rate changed from 0.064 to $0.318 \mathrm{ml} / \mathrm{s}$, the instantaneous output power density decreased from 1200 to $52 \mathrm{~mW} / \mathrm{m}^{2}$, 
while the average power density increased from 5.4 to $16.34 \mathrm{~mW} / \mathrm{m}^{2}$. These results are also due to the increase of amount of water drops and the increase of frequency of contact and removal of water drops on TENG surface.

Figure 6a represents the output $\mathrm{V}_{\mathrm{oc}}$ of $\mathrm{Si}$ solar cell under a room light irradiation (2 $\mathrm{W} / \mathrm{m}^{2}$ ) and water-drop TENG under water dripping at a rate of $0.116 \mathrm{ml} / \mathrm{s}$. It is clearly shown the water-drop TENG can provide a much higher output $\mathrm{V}_{\mathrm{oc}}$ than the Si solar cell. When the fabricated Si solar cell and the rectified TENG device are connected in series, the hybrid cell can harvest both solar energy and rain-drop energy and always provide an electric output in different weather conditions. For practical application in charging a commercial capacitor, the rectified outputs from water-drop TENG and Si solar cell are connected in parallel (inset of Figure 6b). Upon charging a $33 \mu \mathrm{F}$ capacitor, the fabricated solar cell under a light intensity of $1000 \mathrm{~W} / \mathrm{m}^{2}$ was first used and the capacitor can only be charged to $0.6 \mathrm{~V}$ but can not be further charged to higher voltage. Then the rectified TENG under a water dripping rate of $0.116 \mathrm{ml} / \mathrm{s}$ was used to charge the same capacitor, it took $530 \mathrm{~s}$ to charge the capacitor to a voltage of $3.5 \mathrm{~V}$ and the corresponding curves are shown in Figure 6b. This result implies that the water-drop TENG can compensate the disadvantages of solar cell in voltage output. The rectified output of the water-drop TENG is also demonstrated to power 10 commercial green LEDs directly (Figures 6c and 6d). Figure 6e illustrates the dependences of the estimated output power by a water-drop TENG, solar cell and hybrid cell on their surface area under a normal room light irradiation of $2 \mathrm{~W} / \mathrm{m}^{2}$ and a water dripping rate of $0.116 \mathrm{ml} / \mathrm{s}$. Under such room light, the fabricated solar cell can only provide a $\mathrm{V}_{\mathrm{oc}}$ of $0.32 \mathrm{~V}, \mathrm{~J}_{\mathrm{sc}}$ of $0.9 \mathrm{~A} / \mathrm{m}^{2}$ and output power of $0.21 \mathrm{~W} / \mathrm{m}^{2}$, thus we can see that the output performance of solar cell decreased greatly 
compared with the output $\left(160 \mathrm{~W} / \mathrm{m}^{2}\right)$ under a full sun condition. It is worth noting here that a full sun condition is a realistic case because all of the solar panels have to utilize normal sun light at various weather conditions. While the water-drop TENG under a water dripping rate of $0.116 \mathrm{ml} / \mathrm{s}$ can provide an average output power of $16.31 \mathrm{~mW} / \mathrm{m}^{2}$. The theoretical calculation results indicate that water-drop TENG can be a good power source especially in rainy day and night. If the water-drop TENG can be largely applied upon the surface of commercial roof solar panels, then without affecting the output performance of solar cell, a water-drop TENG with surface area of $614 \mathrm{~m}^{2}$ under a dripping rate of $0.116 \mathrm{ml} / \mathrm{s}$ can light up a commercial electricity-saving lamp at a standard horsepower of $10 \mathrm{~W}$. Generally speaking, a separate house in USA often has a roof area more than $1000 \mathrm{~m}^{2}$. If the roof is covered by integrated hybrid cell panels, then in theory, a commercial lamp of $10 \mathrm{~W}$ can be easily lighted up by water impacting in a heavy rainy night. This performance can be largely improved by optimized design and choice of materials. And more importantly, the generated electricity by the water-drop TENG can work together with the solar cells and can be stored in batteries for providing constant electricity output. This result shows great potential of hybrid cell in real industrial applications for green energy harvesting.

\section{Conclusions}

In summary, we have developed a novel hybrid energy cell that consists of a specially processed Si-based solar cell and a special water-drop TENG, which can be used to simultaneously/individually harvest both solar and raindrop electrostatic energy. The bottom solar cell device was designed by using a Si micropyramid $\mathrm{n}^{+}-\mathrm{p}-\mathrm{p}^{+}$junction, which can provide an output voltage of $0.6 \mathrm{~V}$ and output current density of $350 \mathrm{~A} / \mathrm{m}^{2}$ 
under a light intensity of $1000 \mathrm{~W} / \mathrm{m}^{2}$. And the TENG designed for collection of raindrop energy gives an $V_{o c}$ of $30 \mathrm{~V}$ and $J_{s c}$ of $4.2 \mathrm{~mA} / \mathrm{m}^{2}$ when impacted by water drops at a dripping rate of $0.116 \mathrm{ml} / \mathrm{s}$. The transparent TENG can not only be a protection layer of solar cell, but only be a very good complementary energy harvester. The dependence of the output performance of water-drop TENG on incident angles were studied, indicating that the TENG can be applied in widely-used roof solar panels with tilted degree. The relationship between the output power and rain fall precipitation was also studied, showing that a surface area of $614 \mathrm{~m}^{2}$ of hybrid cell can theoretically light up a commercial lamp at a horsepower of $10 \mathrm{~W}$ only by rain dripping. Although the output power is still low, such a hybrid cell paves a new way to simultaneously/individually harvest multimode energies such as solar and raindrop electrostatic energy. As for harvesting green energy from the natural environment, this prototype of hybrid cell has great potential applications in commercial roof solar cell panels in the near future.

\section{Acknowledgement}

This work was supported by U.S. Department of Energy, Office of Basic Energy Sciences (Award DE-FG02-07ER46394), MANA, National Institute For Materials Science, Japan, a joint project with Sungkyunkwan University, Korea, the Hightower Chair foundation, Innovation Program of Shanghai Municipal Education Commission (13YZ104), NSFC (11204172) and the "thousands talents" program for pioneer researcher and his innovation team, China, Beijing City Committee of science and technology ( Z131100006013004, Z131100006013005 ) . 


\section{References}

[1] S. M. Lele, World Dev. 19 (1991) 607-621.

[2] H. Meyar-Naimi, S. Vaez-Zadeh, Energy Policy, 43 (2012) 351-361.

[3] B. Tian, X. Zheng, T. J. Kempa, Y. Fang, N. Yu, G. Yu, J. Huang, C. M. Lieber, Nature 6 (2007) 885-890.

[4] J. H. Oh, H. C. Yuan, H. M. Branz, Nature nanotechnology 7 (2012) 743-748.

[5] P. Devine-Wright, Wind Energy 8 (2005) 125-139.

[6] M. Hoogwijk, A. Faaij, R. van den Broek, G. Berndes, D. Gielen, W. Turkenburg, Biomass and Bioenergy 25 (2003) 119-133.

[7] X. Chen, S. Xu, N. Yao, Y. Shi, Nano Lett. 10 (2010) 2133-2137.

[8] G. Zhu, Z.-H. Lin, Q. S. Jing, P. Bai, C. F. Pan, Y. Yang, Y. S. Zhou, Z. L. Wang, Nano lett. 13 (2013) 847-853.

[9] Y. Yang, W. X. Guo, K. C. Pradel, G. Zhu, Y. S. Zhou, Z. L. Wang, Nano Lett. 12 (2012) 2833-2838.

[10] W. S. Liu, X. Yan, G. Chen, Z. F. Ren, Nano Energy 1 (2012) 42-56.

[11] C. F. Pan, Z. X. Luo, X. Chen, J. Luo, R. R. Liang, G. Zhu, W. Z. Wu, W. X. Guo, X. X. Yan, J. Xu, Z. L. Wang, ACS Nano 5 (2011) 6629-6636.

[12] A. J. Moule, K. Meerholz, Appl. Phys. B - Lasers Opt. 86 (2007) 721-727.

[13] Y. Liu, A. Das, S. Xu, Z. Y. Lin, C. Xu, Z. L. Wang, A. Rohatgi, C. P. Wong, Adv. Energy Mater. 2 (2012) 47-51.

[14] W. L. Min, B. Jiang, P. Jiang, Adv. Mater. 20 (2008) 3914-3918.

[15] S. L. Diedenhofen, G. Vecchi, R. E. Algra, A. Hartsuiker, O. L. Muskens, G. Immink, E. Bakkers, W. L. Vos, J. G. Rivas, Adv. Mater. 21 (2009) 973-978.

[16] F. R. Fan, Z. Q. Tian, Z. L. Wang, Nano Energy 1 (2012) 328-334.

[17] S. H. Wang, L. Lin, Z. L. Wang, Nano Lett.12 (2012) 6339-6346. 
[18] J. Chen, G. Zhu, W. Q. Yang, Q. S. Jing, P. Bai, Y. Yang, Adv. Mater. 25 (2013) 6094-6099.

[19] G. Zhu, J. Chen, T. J. Zhang, Q. S. Jing, Z. L. Wang, Nature communications 5 (2014) 3426:1-9.

[20] G. Cheng, Z.-H. Lin, Z. L. Du, Z. L. Wang, Adv. Funct. Mater. 24 (2014) 28922898.

[21] Y. Yang, H.L. Zhang, J. Chen, S. Lee, T. C. Hou, and Z. L. Wang, Energy \& Environmental Science 6 (2013) 1744-1749.

[22]E. Nemeth, V. Albrecht, G. Schubert, F. Simon, J. Electrostat. 58 (2003) 3-16.

[23] Z.-H. Lin, G. Cheng, L. Lin, S. Lee, Z. L. Wang, Angew. Chem., Int. Ed. 52 (2013) 12545-12549.

[24] Z.-H. Lin, G. Cheng, S. Lee, Z. L. Wang, Adv. Mater. 2014. adma.201400373.

[25] R. Guigon, J.-J. Chaillout, T. Jager, G. Despesse, Smart Mater. Struct. 17(2008) 015039:1-6.

[26] G. Cheng, Z.-H. Lin, Z. L. Du, Z. L. Wang, ACS Nano 8 (2014) 1932-1939.

[27] S. Lee, J. H. Kang, S. J. Lee, W. H. Wang, Lab Chip 9 (2009) 2234-2237.

[28] C. R. Crick, I. P. Parkin, Chem. Commun. 47 (2011) 12059-12061.

[29] S. M. Niu, Y. Liu, S. H. Wang, L. Lin, Y. S. Zhou, Y. F. Hu, Z. L. Wang, Adv. Functional Mater. 24 (2014) 3332-3340.

[30] D. Choi, H. Lee, D. J. Im, I. S. Kang, G. Lim, D. S. Kim, K. H. Kang, K. H. Sci. Rep. 3 (2013) 2037.

[31] B. Ravelo, F. Duval, S. Kane, B. Nsom, J. Electrostat. 69 (2011) 473-478.

[32] T. Paillat, G. Touchard, J. Electrostat. 67 (2009) 326-334.

[33] Y.-M. Chen, C.-H. Lee, H.-C. Wu, Energy Conversion, IEEE Transactions on 20 (2005) 467-473.

[34] A. K. Yadav, S.S. Chandel, Renewable and Sustainable Energy Reviews 23 (2013) 503-513. 


\section{FIGURE CAPTIONS}
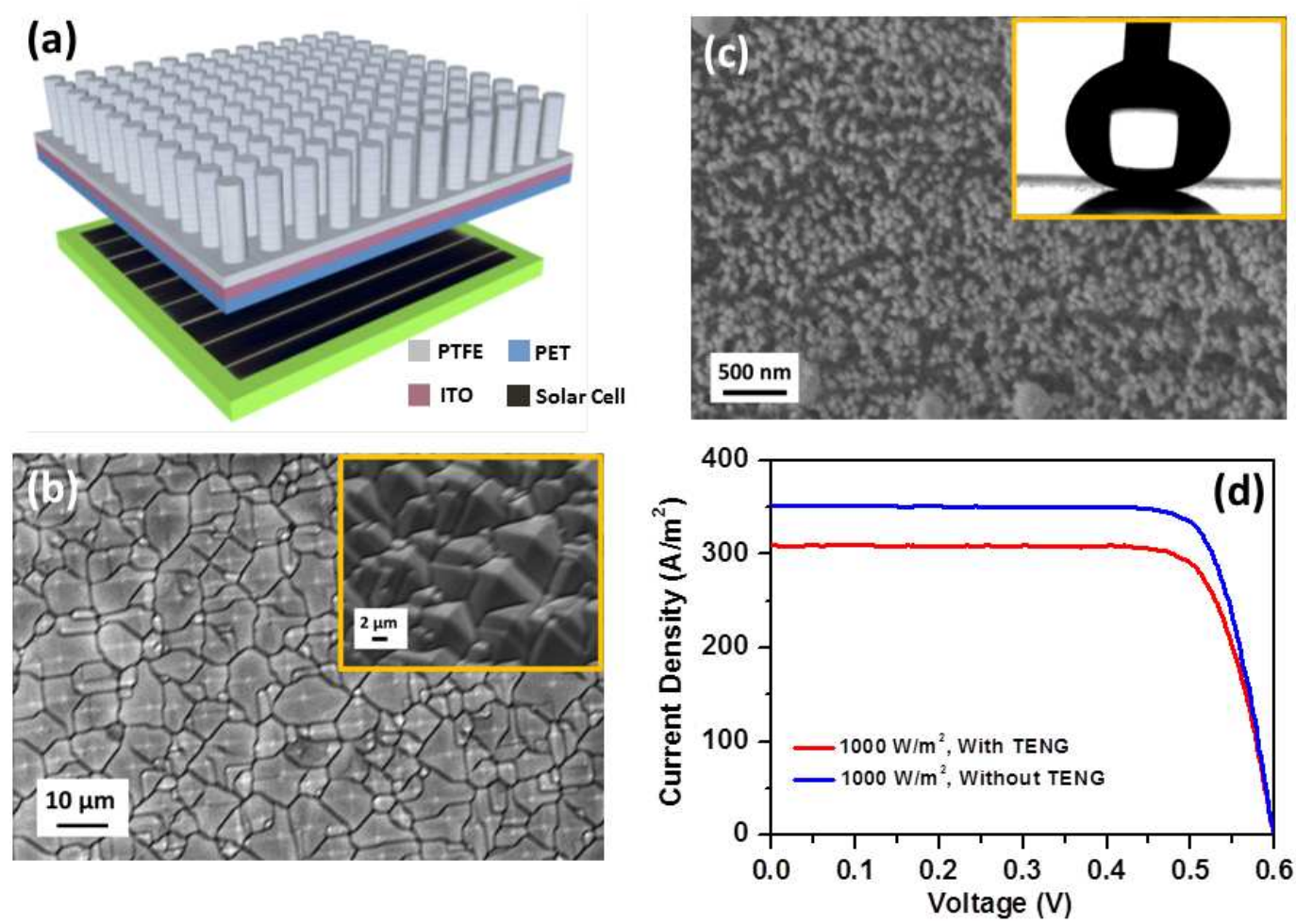

Figure 1. (a) Schematic diagram of the fabricated hybrid energy cell. (b) SEM image of the fabricated silicon-based solar cell surface with micro-/nanostructured pyramids. Inset is a SEM image of the solar cell surface taken from a $20^{\circ}$ tilted angle. (c) SEM image of the PTFE thin film with hierarchical nanostructures. Inset is the incident angle of the PTFE thin film indicating that the film is superhydrophobic. (d) $J$ - $V$ curves of the Si solar cell covered with and without water-drop TENG under the light illumination intensity of $1000 \mathrm{~W} / \mathrm{m}^{2}$. 

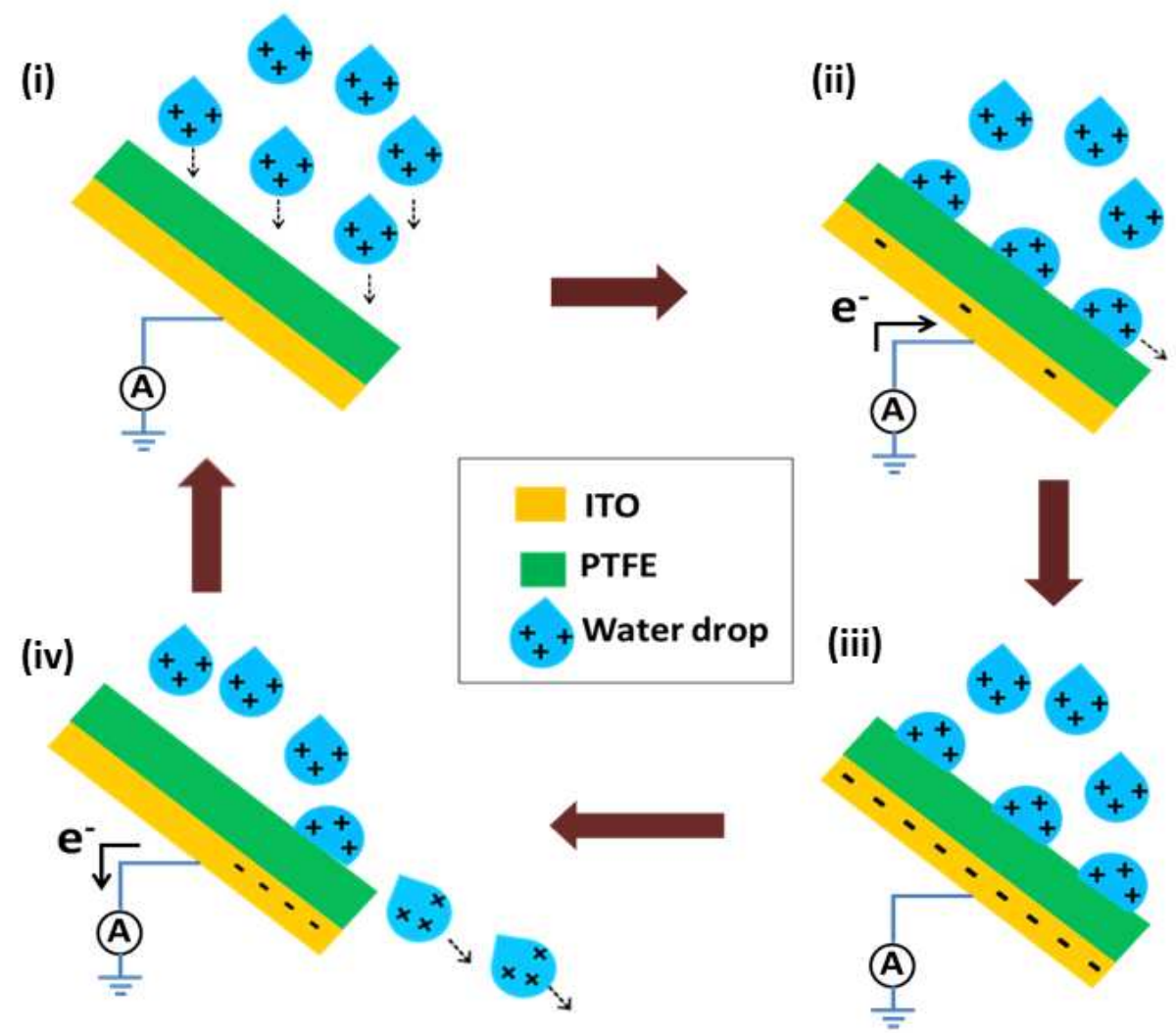

Figure 2. Scheme of the working mechanism of water-drop TENG for water drop electrostatic energy harvesting. The working mechanism is based on a sequential process of the contact electrification and electrostatic induction. 

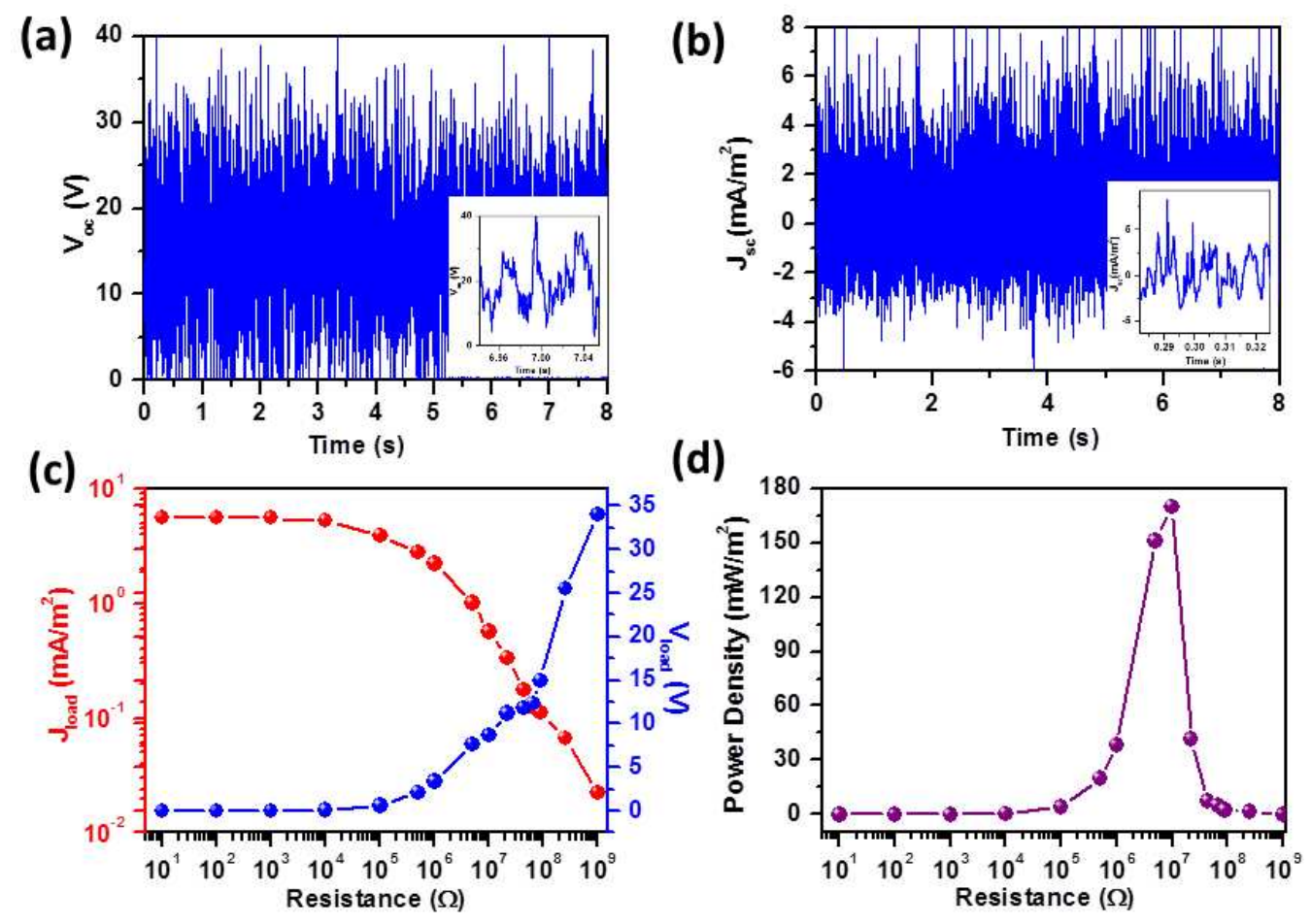

Figure 3. (a) Output voltage and (b) output current density of water-drop TENG for water drop electrostatic energy harvesting. (c-d) The dependences of (c) output voltage (blue), output current density (red) and (d) instantaneous power density (purple) on the resistance of the external load. 
(a)
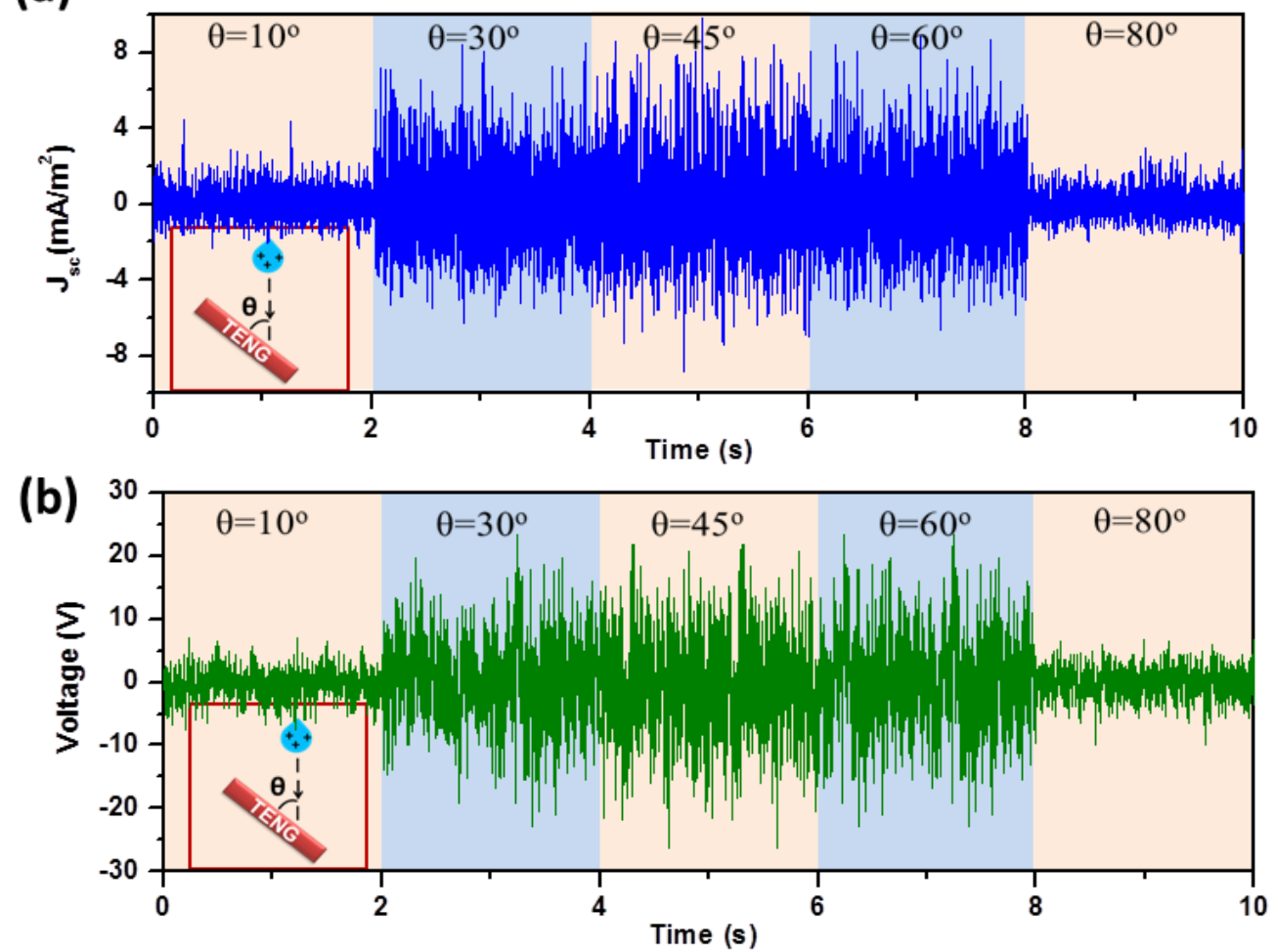

(c)

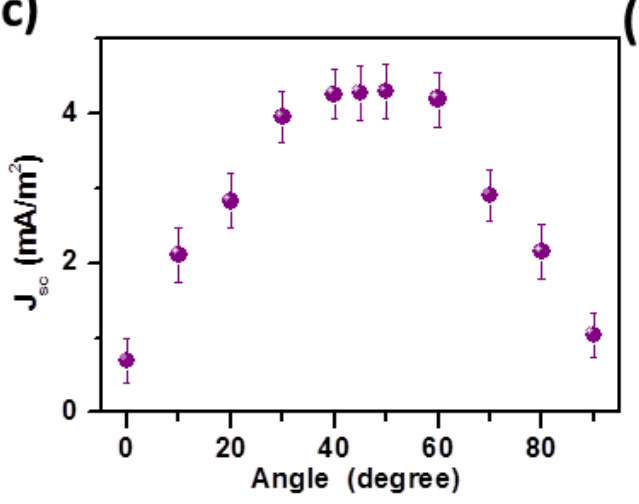

(d)

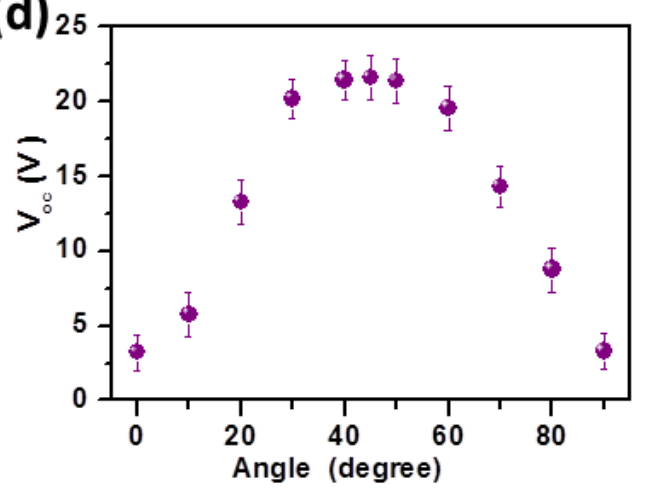

Figure 4. Influence of the incident angle on the output performance of water-drop TENG, where angle $\theta$ is between water dripping direction and the surface of TENG. The measured (a) output current density and (b) output voltage under different $\theta$ from 10 to 80 degree. (c) The summarized relationship between the output current density and the incident angle $\theta$. (d) The summarized relationship between the output voltage and the incident angle $\theta$. 
(a)

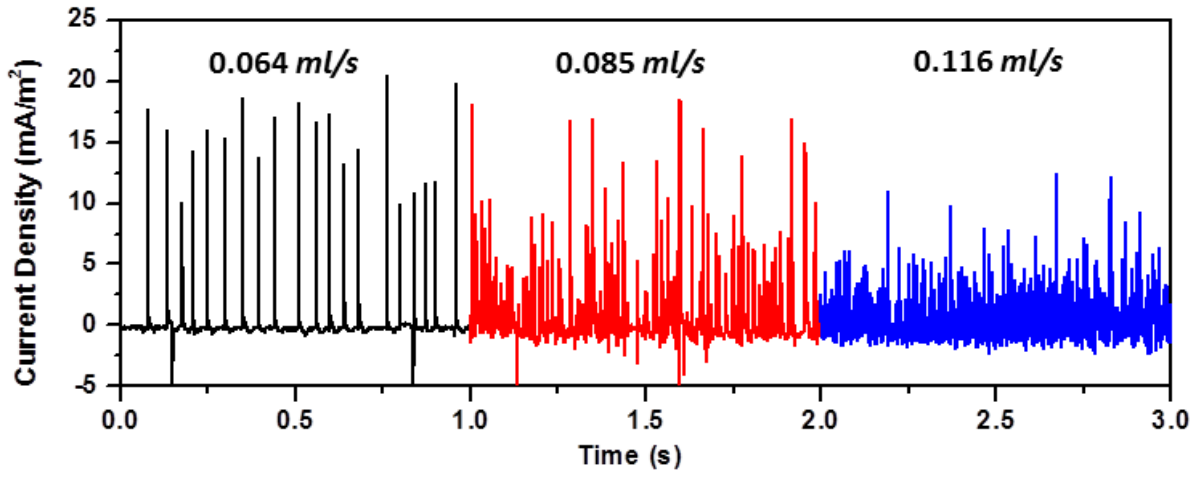

(b)

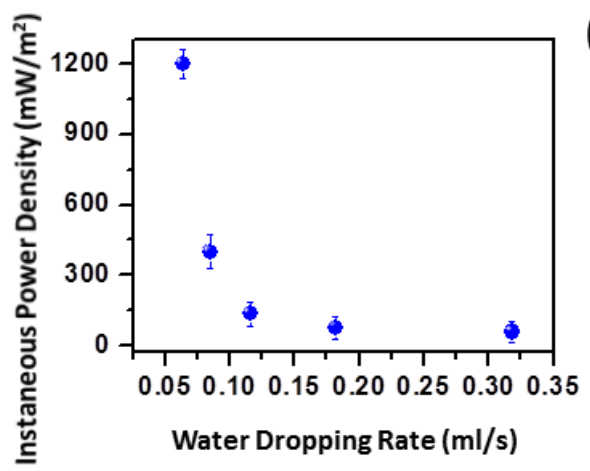

(c)

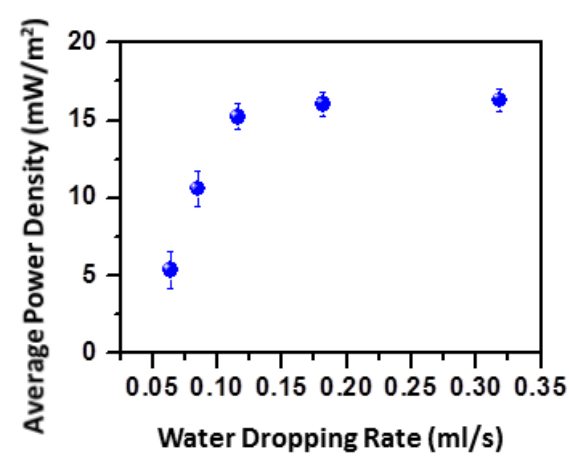

Figure 5. (a) The output current density of water-drop TENG generated by water drops at different dripping rates. The dependences of (b) instantaneous output power density and (c) average output power density of TENG on water dripping rate. 

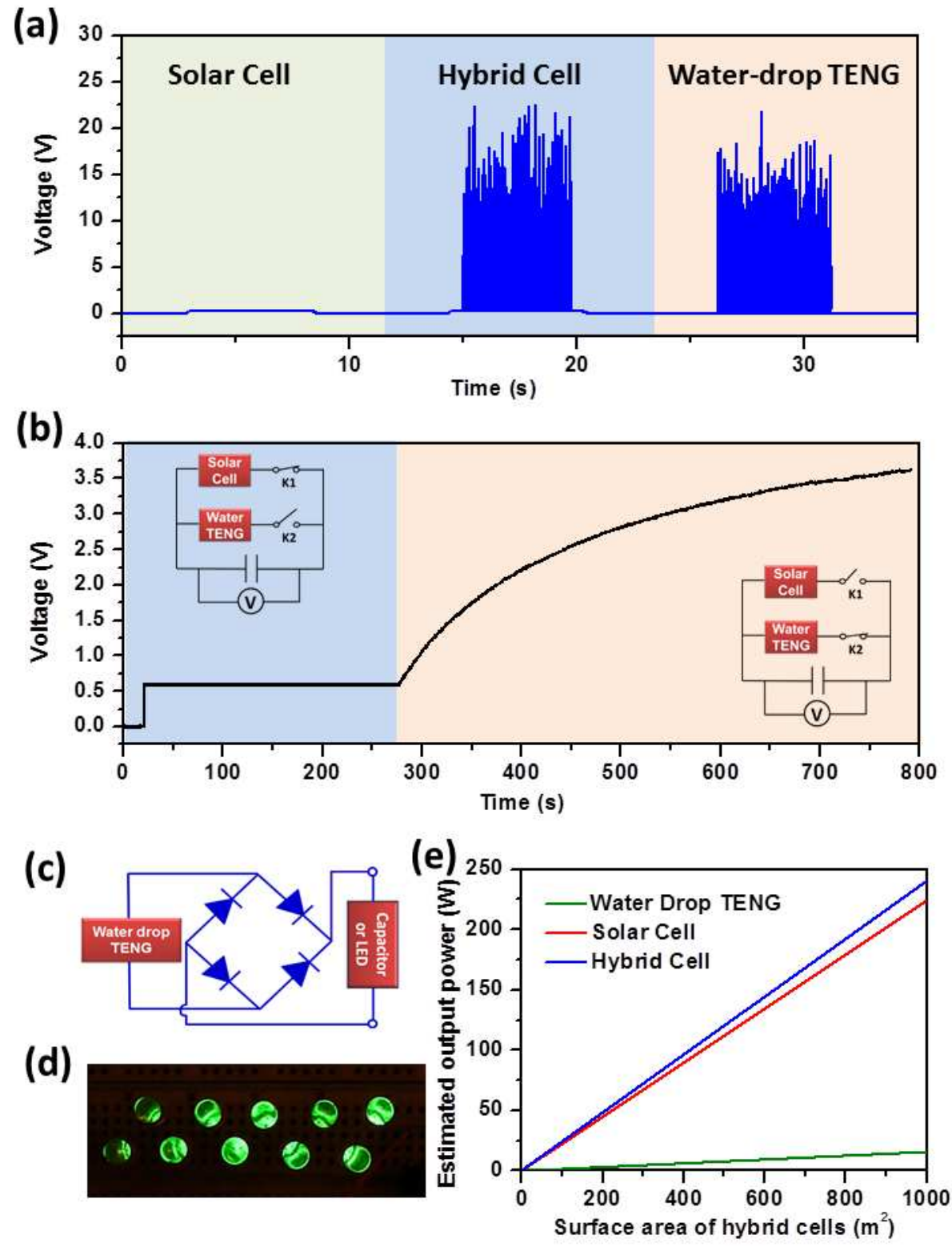

Figure 6. (a) Output voltage of the fabricated Si solar cell, hybrid cell, and water-drop TENG. (b) The measured voltage of a $33 \mu \mathrm{F}$ capacitor charged by the solar cell and water-drop TENG consequently. (c) The diagram of the rectifying circuit used by TENG to charge LED bulbs or to charge a capacitor. (d) The photography of 10 commercial LED bulbs driven by water-drop TENG at a dripping rate of $0.116 \mathrm{~m} \square / \mathrm{s}$. (e) Dependences of estimated output power of water-drop TENG, solar cell and hybrid cell on their surface area under normal room light irradiation $\left(2 \mathrm{~W} / \mathrm{m}^{2}\right)$. 
Vitae

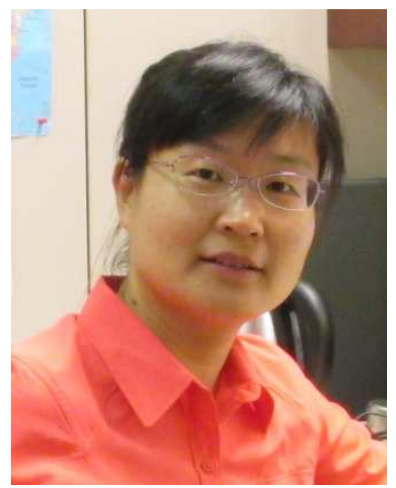

Li Zheng is a visiting scholar in School of Materials Science and Engineering at Georgia Institute of Technology and an assistant professor in Shanghai University of Electric Power. She received her B. S. in physics from Ocean University of China in 2002, M.S. in optics from Shanghai Institute of Optics and Fine Mechanics, Chinese Academy of Sciences in 2006 and Ph. D. in Optics from Shanghai Jiao Tong University in 2009. Her current research interests include nanowire lasers, nanostructure-based optoelectronic devices, nanogenerator, and self-powered nanosensors.

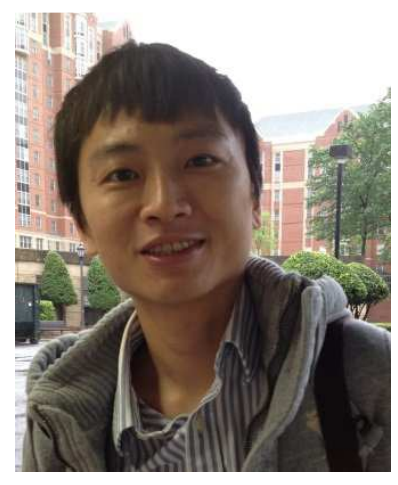

Zong-Hong Lin received his Ph.D degree from Department of Chemistry, National Taiwan University in 2009. Before joining the Institute of Biomedical Engineering, National Tsing Hua University in Taiwan as an assistant professor, he was a postdoctoral researcher supervised by Professor Zhong Lin Wang in the school of Material Science and Engineering, Georgia Institute of Technology. His research interests include the development of high-output generators with novel design for efficient energy harvesting, self-powered systems for biomolecules detection and environmental sensors, highly efficient and stable catalysts for fuel cell applications, and fabrication of metal/semiconductor nanowires. 


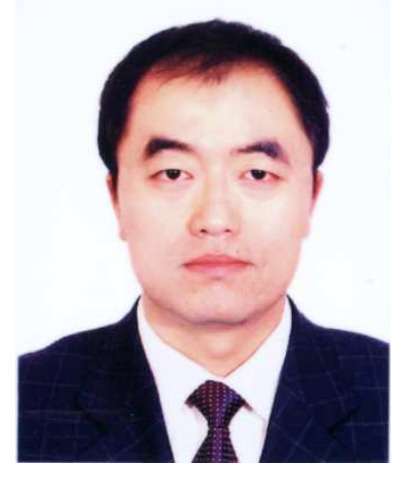

Gang Cheng is a visiting scholar in School of Materials Science and Engineering at Georgia Institute of Technology and an associate professor in Henan University. He received the B. S. degree from Henan University in 2000 and the Ph. D. degree from Jilin University in 2008. His research interests are nanostructure-based electronic and optoelectronic devices, nanogenerator, and self-powered nanosensors.

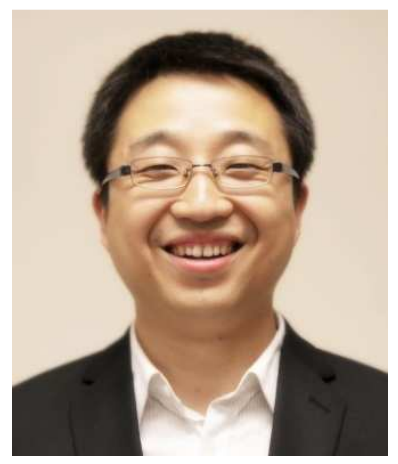

Wenzhuo Wu is a postdoctoral fellow in School of Materials Science and Engineering at Georgia Institute of Technology. He received his B.S. in Electronic Information Science and Technology in 2005 from the University of Science and Technology of China (USTC), Hefei and his M. Eng. in Electrical and Computer Engineering from the National University of Singapore (NUS) in 2008. After working at Chartered Semiconductor Manufacturing (now GlobalFoundries) in Singapore from 2007 to 2008, he began the doctoral research under the supervision of Prof. Zhong Lin Wang and received his Ph. D. from Georgia Institute of Technology in Materials Science and Engineering in 2013. Wenzhuo's research interests include synthesis, fabrication and integration of nanomaterials/devices; nanotechnology-enabled applications in energy harvesting/conversion/storage, electronics, optoelectronics, sensing and interfacing; piezotronics/piezo-phototronics; self-powered micro/nano-systems. 


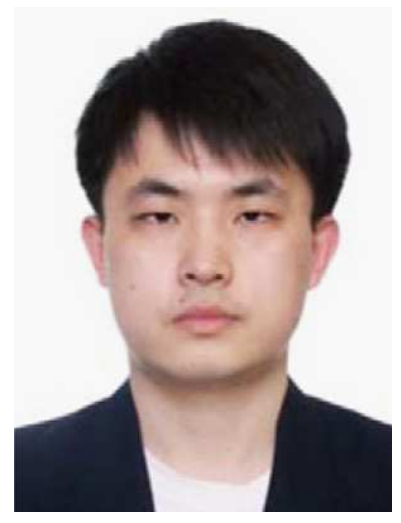

Xiaonan Wen received his B.S. degree in Physics from Peking University, China in 2010. $\mathrm{He}$ is currently a Ph.D. candidate in the School of Materials Science \& Engineering at Georgia Institute of Technology. His research interests include synthesis of functional nanomaterials, energy harvesting using piezoelectric and triboelectric generators, selfpowered nanosystems, piezo-electronics and piezo-optoelectronics based on $\mathrm{ZnO}, \mathrm{GaN}$ etc. for novel transistors, devices and integration of them into functional systems.

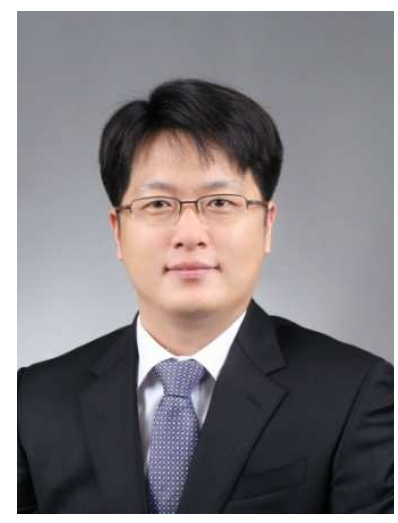

Sangmin Lee received his Ph.D. in Mechanical Engineering from Pohang University of Science and Technology (POSTECH) in 2011. Now he is an assistant professor in the School of Mechanical Engineering at Chung-Ang University. His main research interests focus on the fields of piezoelectric and triboelectric nanogenerator for energy harvesting device and self-powered active sensor, super-flexible piezoelectric nanogenerator for self-powered/active sensor, hybrid cell for simultaneously harvesting multiple types of energies, surface wetting control including superhydrophobicity/superhydrophilicity based on micro/nanofabrications and chemical treatment, mechanical characterization of micro/nanostructured surface. 


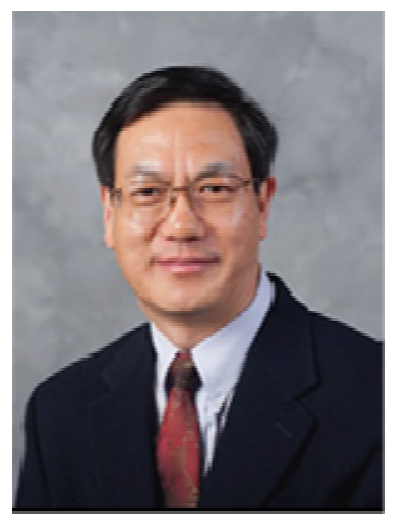

Zhong Lin (ZL) Wang received his Ph.D. from Arizona State University in physics. He now is the Hightower Chair in Materials Science and Engineering, Regents' Professor, Engineering Distinguished Professor and Director, Center for Nanostructure Characterization, at Georgia Tech. Dr. Wang has made original and innovative contributions to the synthesis, discovery, characterization and understanding of fundamental physical properties of oxide nanobelts and nanowires, as well as applications of nanowires in energy sciences, electronics, optoelectronics and biological science. His discovery and breakthroughs in developing nanogenerators established the principle and technological road map for harvesting mechanical energy from environment and biological systems for powering a personal electronics. His research on self-powered nanosystems has inspired the worldwide effort in academia and industry for studying energy for micro-nano-systems, which is now a distinct disciplinary in energy research and future sensor networks. He coined and pioneered the field of piezotronics and piezophototronics by introducing piezoelectric potential gated charge transport process in fabricating new electronic and optoelectronic devices. Details can be found at: http://www.nanoscience.gatech.edu/. 


\section{*Graphical Abstract}

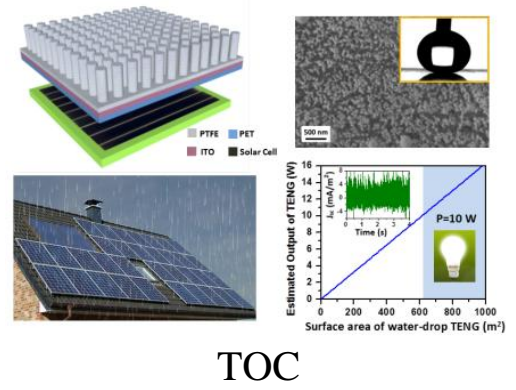

(1) TOC

.

